\title{
Curva nacional de distribución de peso al nacer según edad gestacional. Chile, 1993 a 2000
}

\author{
A national birth weight distribution \\ curve according to gestational age in \\ Chile from 1993 to 2000
}

$\mathrm{S}_{\mathrm{s}}^{\mathrm{r}}$ Editor: Me refiero al artículo "Curva nacional de distribución de peso al nacer según edad gestacional. Chile, 1993-2000", de González y otros $^{1}$, en el que se menciona erróneamente el nombre de este Departamento, al describir la base de datos de nacimientos vivos (no la del Registro Civil completa, como ahí se sugiere) que nosotros mismos pusimos a disposición de los investigadores, a quienes apoyamos además en su trabajo durante un prolongado período.

En la página 1156, bajo el subtítulo "Materiales y métodos" se sugiere que "la base de datos del Servicio de Registro Civil" estaría "compartida por el Instituto Nacional de Estadísticas y el Departamento de Informática de este Ministerio", lo que es completamente erróneo, toda vez que la instalación del acceso en línea a los registros de nacimientos, defunciones y defunciones fetales del Sistema de Información del Servicio de Registro Civil e Identificación es una obra total y completa de este Departamento, que representa al Ministerio de Salud en el Convenio Tripartito vigente entre las tres instituciones. No ha habido ingerencia de ningún otro organismo ministerial en este proceso de modernización.

Considero que un Comité Editorial debería preocuparse de esa clase de detalles en sus publicaciones, aparte de otros, que revelan un escaso manejo de conceptos básicos de indicadores de salud, como por ejemplo, que en el párrafo anterior al citado, se habla de "las mortalidades perinatal e infantil en 2002, de 8,6\% y $8,9 \%$ por mil recién nacidos vivos". ¿Qué estaría ocurriendo en Chile si tuviéramos esa mortalidad infantil?

\section{Dra. Danuta Rajs}

Jefe Departamento Estadísticas e Información de Salud, Ministerio de Salud

\section{REFERENCIA}

1. González R, Gómez R, Castro R, Kae J, Merino P, ETCHEGARAY A ET AL. Curva nacional de distribución de peso al nacer según edad gestacional. Chile, 1993 a 2000. Rev Méd Chile 2004; 132: 1155-65. 
RÉPLICA DE LOS AUTORES ALUDIDOS

Q r Editor: En relación a la carta de la Dra. SDanuta Rajs, Jefe Departamento Estadísticas e Información de Salud, Ministerio de Salud, sobre el artículo "Curva Nacional de distribución de peso al nacer según edad gestacional. Chile 1993 a 2000" (publicado en el número de octubre 2004) del cual los firmantes somos autores, nos interesa precisar lo siguiente:

1. En primer lugar expresar los agradecimientos a la labor de la Dra. Rajs y de su equipo, sin su trabajo y apoyo ninguno de estos estudios de la situación nacional materno infantil serían posibles. Precisamente la validez de este estudio está basado en la calidad de su trabajo. El análisis de esta información ha sido clave en la discusión y planificación de las políticas en nuestro sector.

2. Es absolutamente correcto lo que menciona la Dra. Rajs sobre la Sección de Materiales y
Método, es decir la fuente original de datos para este y otros estudios ha sido el Departamento de Estadísticas e Información de Salud del Ministerio de Salud.

3. Lamentamos al igual que la Dra. Rajs el error tipográfico expresado en la introducción de nuestro manuscrito, donde debiera expresarse que las mortalidades perinatal e infantil en 2002, han sido de 8,6 y 8,9 por mil recién nacidos vivos, respectivamente (no \%).

Le saludan atentamente,

\section{Dr. Rogelio González P.}

Unidad de Alto Riesgo Obstétrico

Hospital Sótero del Río

\section{Dr. René Castro S.}

Departamento de Salud de la Mujer Ministerio de Salud de Chile

Nota del Editor: La Revista Médica de Chile publicó en su número de marzo de 2005 una Fe de Erratum, corrigiendo la tasa de mortalidad perinatal. Las pruebas de impresión de este artículo habían sido revisadas, entre otros, por uno de los autores. 